\title{
Fatores de risco para desenvolvimento de diabetes mellitus do tipo 2 em estudantes universitários do curso de enfermagem do interior do Amazonas
}

\author{
Risk factors for development of type 2 diabetes mellitus in university students in the \\ Amazon internal nursing course
}
Factores de riesgo para el desarrollo del tipo 2 diabetes mellitus en estudiantes universitários en el curso de enfermería interna de Amazonia

Vanderson de Souza Pereira ${ }^{1 *}$, Deyvylan Araujo Reis², Stefany Alencar de Oliveira.

\section{RESUMO}

Objetivo: identificar os fatores de risco para o desenvolvimento de Diabetes Mellitus tipo 2 em estudantes universitários do Curso de Enfermagem. Método: estudo exploratório, descritivo e transversal, com abordagem quantitativa, realizado com 154 universitários do Curso de Enfermagem, Coari, Amazonas, Brasil. Os dados foram coletados por meio de dois instrumentos, o questionário sociodemográfico e o instrumento validado e adaptado chamado de "Are you at risk for type 2 diabetes?". A análise dos dados foi realizada por meio de estatística descritiva e inferencial, utilizando o Microsoft Excel e o SPSS versão 20.0. Resultados: houve predomínio do sexo feminino $(68,2 \%)$, com média de idade de $23,1( \pm 4,3)$ anos e de solteiros $(78,6 \%)$. A maioria faz consumo adequado de sal $(53,9 \%)$, não tem hipertensão arterial sistêmica $(94,2 \%)$ e considera a própria saúde boa $(55,8 \%)$, não possui casos de Diabetes Mellitus na família $(54,5 \%)$, nem risco $(98,0 \%)$ para o desenvolvimento da doença. Conclusão: desta forma, esta investigação constatou que, apesar de ocorrer uma transição no estilo de vida do estudante ao ingressar na vida acadêmica, isso não influencia significativamente o desenvolvimento de Diabetes Mellitus tipo 2.

Palavras-chave: Fator de risco; Diabetes Mellitus; Enfermagem.

\begin{abstract}
Objective: To identify the risk factors for the development of type 2 Diabetes Mellitus in university students of the Nursing course. Method: Exploratory, descriptive and transverse study with quantitative approach, accomplished with 154 academics from the Nursing course, Coari, Amazonas, Brazil. Data were collected through two instruments, the sociodemographic questionnaire and the validated and adapted instrument called "Are you at risk for type 2 diabetes?". Data analysis was performed using descriptive and inferential statistics using Microsoft Excel and SPSS version 20.0. Results: there was a predominance of females (68.2\%), with a means age of 23.1 ( \pm 4.3 ) years and single $(78.6 \%)$. Most have adequate salt intake $(53.9 \%)$, do not have systemic arterial hypertension (94.2\%) and consider their own health good (55.8\%), have no cases of diabetes mellitus in the family $(54.5 \%)$, nor risk $(98.0 \%)$ for the development of the disease. Conclusion: Thus, this investigation found that, although there is a transition in the student's lifestyle upon entering academic life, this does not significantly influence the development of Type 2 Diabetes Mellitus.
\end{abstract}

Keywords: Risk factor; Diabetes Mellitus; Nursing.

1Universidade Federal do Amazonas (UFAM), Coari-Amazonas. *E-mail: vanderson_spereira@outlook.com

SUBMETIDO EM: 6/2019

ACEITO EM: 7/2019

PUBLICADO EM: 8/2019 


\section{RESUMEN}

Objetivo: Identificar los factores de riesgo para el desarrollo de Diabetes Mellitus tipo 2 en estudiantes universitarios del curso de Enfermería. Método: estudio exploratorio, descriptivo y transversal, de abordaje cuantitativo, realizado con 154 universitarios del curso de Enfermería, Coari, Amazonas, Brasil. Los datos fueron recolectados por medio de dos instrumentos, un cuestionario sociodemográfico y un instrumento adaptado y validado llamado “¿Are you at risk for type 2 diabetes?". El análisis de los datos fué realizado por medio de estadística descriptiva e inferencial, utilizando los programas Microsoft Excel y SPSS versión 20.0. Resultados: El sexo femenino fue predominante $(68,2 \%)$, con uma media de edad de $23,1( \pm 4,3)$ años, igual que los solteros $(78,6 \%)$. La mayoría tiene un consumo adecuado de sal $(53,9 \%)$, no tiene hipertensión arterial sistémica $(94,2 \%)$ y considera que su propia salud es buena $(55,8 \%)$, no tiene casos de Diabetes Mellitus en la familia $(54,5 \%)$, ni riesgo para el desarrollo de la enfermedad $(98,0 \%)$. Conclusión: De esta forma, esta investigación demostró que, a pesar de que ocurre una transición en el estilo de vida del estudiante al ingresar a la vida académica, esto no ejerce una influencia significativa en el desarrollo de la Diabetes Mellitus tipo 2.

Palabras clave: Factor de riesgo; Diabetes Mellitus; Enfermería.

\section{INTRODUÇÃO}

As Doenças Crônicas Não-Transmissíveis (DCNTs) são um fenômeno que vem ocorrendo nas últimas décadas em todos os países, ocasionando grandes impactos na mortalidade, morbidade e no custo decorrente da assistência de saúde. No ano de 2008, já se registrava que o número total de óbitos ocorrido no mundo era de 63\% para as DCNTs (DUNCAN BB et al., 2012).

No Brasil, as DCNTs são um problema de saúde de maior magnitude e foram responsáveis por cerca de $74,6 \%$ das causas de mortes no ano de 2013 , atingindo fortemente as camadas pobres da população e grupos vulneráveis. Essas enfermidades foram atribuídas às doenças cardiovasculares, ao câncer, ao diabetes e às doenças respiratórias crônicas (BRASIL, 2011; SCHMIDT MI et al., 2011).

Entre as DCNTs, a Diabetes Mellitus (DM) aparece no grupo de doenças como quarta causa de mortalidade no Brasil e sexta no mundo, sendo caracterizado como um grupo de doenças metabólicas resultantes na hiperglicemia, devido à alteração na secreção de insulina ou em sua ação no organismo (MALETTA CHM, 2016).

Há vários fatores de risco para DM do tipo II como: doenças cardiovasculares, síndrome de ovários micropolicísticos, tolerância à glicose diminuída e hemoglobina glicada igual ou superior a 5,7\%, além de idade, sexo, hereditariedade, sobrepeso, sedentarismo, diabetes gestacional, hipertensão arterial, entre outros (MARINHO N et al., 2013; SUDARTH BE et al., 2016).

No ano de 2015, foram registradas 14,3 milhões de pessoas com DM no Brasil, na faixa etária entre 20 e 79 anos, podendo alcançar 23,3 milhões no ano de 2040. A DM é uma epidemia que está em curso, nos dias atuais, e estima-se que, no cenário mundial, o número de pessoas diagnosticadas com DM atinja a ordem de 415 milhões de habitantes e que, até 2040, esse número suba para 642 milhões, bem como que cerca de 193 milhões de pessoas ainda não tenham sido diagnosticadas (IDF, 2015).

Ao ingressar na universidade, o estudante passa por diversas mudanças, como os hábitos de vida e alimentares, a diminuição nas atividades físicas e horas dedicadas aos estudos, o que resulta no sedentarismo e sobrepeso. Cita-se também outro fator que tem contribuído, a evolução tecnológica, que ajuda a minimizar o esforço físico nas atividades cotidianas e com isso pode favorecer o aparecimento de um ou vários fatores de risco para desenvolver DM tipo 2 (LIMA ACS et al., 2014; MAGALHÃES AT et al., 2015).

Devido à crescente taxa de incidência da DM que vem acometendo a população nas últimas décadas e que tem ganhado proporções alarmantes no cenário mundial, e ao impacto causado no âmbito psicossocial dos indivíduos acometidos, além dos danos físicos através da complicação dessa DCNT que se tornam 
irreparáveis, despertou-se o interesse em realizar esta pesquisa. Portanto este estudo pode contribuir no planejamento de ações de prevenção por parte dos profissionais de saúde da Estratégia Saúde da Família e da própria Instituição de Ensino, a fim de realizar abordagem na promoção de saúde de forma mais efetiva voltada aos estudantes universitários.

Nesse contexto, percebe-se a importância do estudo em questão, o que torna imprescindível o conhecimento como forma para possibilitar o desenvolvimento de políticas públicas e subsidiar as ações de saúde desenvolvidas em serviço de saúde na atenção primária, principalmente na prevenção e promoção da saúde com esse público-alvo.

Diante do exposto, surge o seguinte questionamento do estudo: Quais são os fatores de risco para o desenvolvimento de DM do tipo 2 em estudantes universitários do curso de enfermagem do Instituto de Saúde e Biotecnologia da Universidade Federal do Amazonas? Por conseguinte, o objetivo desta investigação foi identificar os fatores de risco para o desenvolvimento de Diabete Mellitus tipo 2 prevalentes em estudantes universitários do curso Enfermagem.

\section{MÉTODOS}

Trata-se de um estudo exploratório, descritivo e transversal, com abordagem quantitativa, realizado em uma universidade pública federal, localizada no município de Coari do Estado do Amazonas, Brasil. Foram selecionados universitários do Curso de Enfermagem, totalizando 154 acadêmicos do segundo, quarto, sexto, oitavo e décimo períodos, totalizando todos os alunos matriculados nesses períodos. Para a obtenção da população do estudo, os pesquisadores solicitaram da Coordenação Acadêmica da Universidade a relação de todos os alunos efetivamente matriculados no Curso de Enfermagem através do Sistema de Informação para o Ensino (SIE), principalmente os discentes com idade igual ou superior a 18 anos.

O período de coleta de dados foi de abril a maio de 2018, em dias e horários aleatórios. O procedimento de coleta consistiu no contato inicial com a coordenadora acadêmica e os professores para obter informações sobre os alunos matriculados e programar um dia e horário para apresentar a pesquisa aos estudantes. No segundo momento, em sala de aula, após a autorização do professor, ocorreu uma breve explicação pelos pesquisadores aos alunos sobre a pesquisa, enfatizando os objetivos do estudo, os benefícios e riscos da participação no estudo. Após a explicação, os pesquisadores convidaram os acadêmicos para participar do estudo, e aqueles que concordavam em participar, assinavam o Termo de Consentimento Livre e Esclarecido (TCLE). Os questionários foram entreguem àqueles estudantes que aceitaram participar do estudo e estes foram orientados a preenchê-los corretamente e a fazer a devolução no mesmo dia, levando em torno de 20 minutos para responder aos instrumentos de coleta de dados (autoaplicáveis).

Incluíram-se na investigação os participantes que se enquadraram nos seguintes critérios: estudantes de graduação em enfermagem, com idade mínima de 18 anos, de ambos os sexos, matriculados. Foram excluídos indivíduos com diagnóstico médico ou autorrelato de DM e gestantes.

Para a coleta de dados, foram utilizados dois instrumentos, tendo sido um construído pelos pesquisadores e outro já adaptado e validado no Brasil. O primeiro consistiu no questionário com 36 questões (contendo alternativas como opção de escolha nas questões que o participante poderia assinalar), atendendo às variáveis demográfica, socioeconômica, vida acadêmica, domiciliar, saúde e comportamental.

Destaca-se que, no questionário, foram utilizadas algumas questões sobre as variáveis demográfica e socioeconômica do instrumento da Amostra por Domicílio do Censo Demográfico do ano de 2010, do Instituto Brasileiro de Geografia e Estatística (IBGE, 2012) e, para as variáveis da vida acadêmica, utilizou-se o questionário do Instituto Nacional de Estudos e Pesquisas Educacionais Anísio Teixeira (INEP, 2016) para estudantes do ano de 2015.

Para as variáveis comportamentais (hábito alimentar e de vida), foram utilizadas algumas questões adaptadas do instrumento do Programa Vigilância de Fatores de Risco e Proteção para Doenças Crônicas por Inquérito Telefônico do ano de 2015 (Vigitel) (BRASIL, 2016). Depois da construção do questionário, este 
foi submetido ao pré-teste com sete estudantes, e concluiu-se não haver necessidade de realizar alterações no instrumento.

Em seguida, foi aplicado o questionário chamado de "Are you at risk for type 2 diabetes?", buscando verificar o risco de desenvolver DM tipo 2, tendo sido classificado com risco aqueles que obtivessem escore maior ou igual a cinco. Os dados e as informações coletadas foram registrados em uma planilha do programa Microsoft Excel 2010, para análise estatística posterior, que utilizou o programa Statistical Package For The Sciences (SPSS versão 20.0), mediante análise descritiva dos dados, procedendo das variáveis do estudo, por meio da distribuição de frequência absoluta e relativa, além das medidas descritivas (média, desviopadrão). Para verificar a existência de associação entre as variáveis, utilizou-se o teste Qui-quadrado de Pearson e Exato de Fisher, sendo adotado um nível de significância de $5 \% \quad(p<0,05)$. Para facilitar a apresentação dos dados, foram elaboradas tabelas e gráficos. Em observância aos aspectos éticos da pesquisa em seres humanos, preconizados pelo Conselho Nacional de Saúde (CNS), na Resolução no 466/2012, o projeto foi aprovado pelo Comitê de Ética em Pesquisa (CEP) da Universidade Federal do Amazonas, processo ㄲo 2.418 .606 e no de CAAE 78340517.1.0000.5020.

\section{RESULTADOS E DISCUSSÃO}

Os dados sociodemográficos são descritos na Tabela 1.

Tabela 1 - Dados sociodemográficos dos estudantes do Curso de Enfermagem do Instituto de Saúde e Biotecnologia da Universidade Federal do Amazonas. Coari, Amazonas, Brasil, 2018.

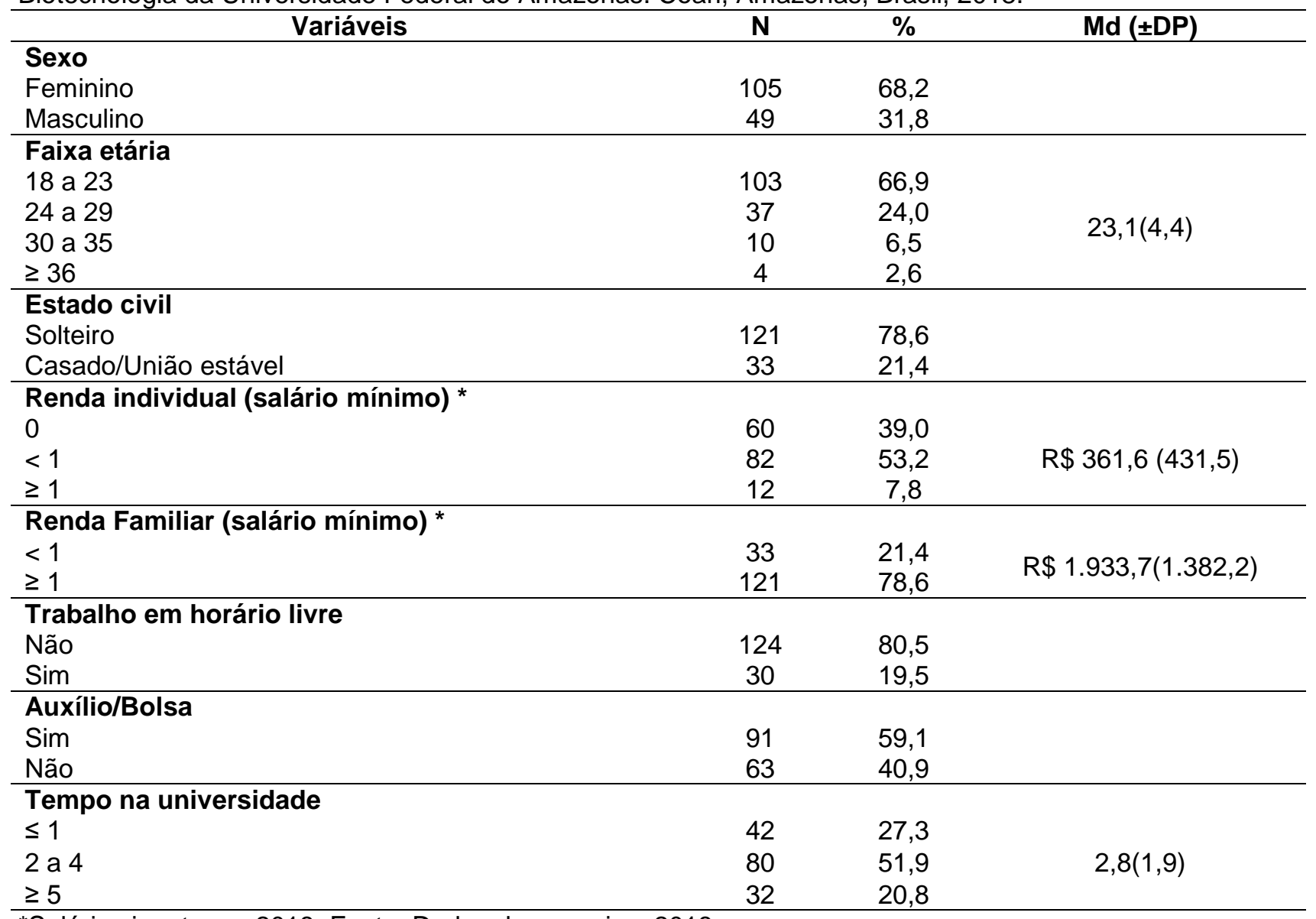

*Salário vigente em 2018. Fonte: Dados da pesquisa, 2018.

Quanto ao sexo, foi constatado maior predominância para o gênero feminino, o que corrobora os resultados apresentados em outras publicações, Bublitz S (2016), Barlem JGT (2014), 84,5\% e 92,9\% respectivamente. $O$ perfil do profissional enfermeiro é historicamente feminino e se dá a partir de Florence 
Nightingale, da qual foi herdada a possibilidade de a mulher sair da esfera privada para a vida pública (SEHNEM D et al., 2013). O estudo de Crepaldi BVC et al. (2016) realizado com alunos de uma universidade pública do município de Uberlândia, Minas Gerais, Brasil, apresentou certo equilíbrio entre os participantes com relação ao sexo, uma vez que $51,7 \%$ dos alunos eram mulheres.

Para a variável idade, os dados foram organizados em quatro faixas. Observaram-se as maiores frequências na primeira faixa etária, 18 a 23 anos (66,9\%). Para essa variável, a média é igual a $23,1(\mathrm{dp}=4,4)$ anos, o que evidencia que, com relação à idade, a maioria dos estudantes universitários do curso de enfermagem são jovens, o que é comprovado a partir dos resultados nas publicações de Santos JDS et al. (2015) e Gonçalves A et al. (2016), nos quais a média de idade encontrada foi 23,4 e 22,4 anos respectivamente. Em relação à cor/raça, a maioria dos participantes da pesquisa são pardos $(78,6 \%)$. No que tange à cidade de origem dos estudantes universitários, quase a metade é do município de Coari $(47,4 \%)$, sede do presente estudo. Quanto à cor/raça parda, este dado encontra-se com valor alto em relação à frequência apresentada pelo censo demográfico 2010 realizado pelo Instituto Brasileiro de Geografia e Estatística (IBGE, 2012), para o Estado do Amazonas (68,9\%). A investigação de Castro IPP et al. (2015), realizada em uma universidade privada no município de São Luís do Estado de Maranhão, com 52 acadêmicos, mostrou que a maior parte dos participantes eram brancos $(42,0 \%)$, o que contrasta com a população do estado e também desta pesquisa, uma vez que o censo de 2010 apresenta uma predominância da cor parda $(66,5 \%)$.

Mais da metade dos estudantes do presente estudo se encontram periodizados (58,4\%), e os que estão no primeiro curso superior totalizam $90,3 \%$. A maior parte afirma não ter uma boa qualidade de sono durante o semestre na universidade (81,8\%). Lourenço CLM et al. (2016), ao inquirirem sobre o comportamento sedentário, com 1.085 universitários em uma universidade do Estado da Bahia, afirmam que, quando questionados sobre a autopercepção de sono, 51,5\% consideram positiva, 35,2\% intermediária e apenas $13,3 \%$ têm uma autopercepção de sono negativa.

\section{Características de hábitos alimentares e de vida}

A Tabela 2 apresenta os alimentos consumidos semanalmente pelos acadêmicos de enfermagem.

Tabela 2 - Frequência do hábito alimentar em estudantes do Curso de Enfermagem do Instituto de Saúde e Biotecnologia da Universidade Federal do Amazonas. Coari, Amazonas, Brasil, 2018.

\begin{tabular}{lccccc}
\multicolumn{1}{c}{ Variáveis } & \multicolumn{5}{c}{ Frequência por semana } \\
\hline Grupos Alimentos & 1 a 2 & 3 a 4 & 5 a 6 & Todos os dias & Não consomem \\
\hline Alimentos Saudáveis & $\mathbf{N}(\%)$ & $\mathbf{N}(\%)$ & $\mathbf{N}(\%)$ & $\mathbf{N}(\%)$ & $\mathbf{N}(\%)$ \\
Consumo de feijão & $50(32,5)$ & $38(24,7)$ & $24(15,6)$ & $20(13,0)$ & $22(14,3)$ \\
Consumo de verduras e legumes & $23(14,9)$ & $43(27,9)$ & $20(13,0)$ & $46(29,9)$ & $22(14,3)$ \\
Consumo de carne & $33(21,4)$ & $69(44,8)$ & $35(22,7)$ & $2(1,3)$ & $15(9,7)$ \\
Consumo de frango/galinha & $15(9,7)$ & $66(42,9)$ & $53(34,4)$ & $15(9,7)$ & $5(3,3)$ \\
Consumo de suco de frutas natural & $33(21,4)$ & $42(27,3)$ & $29(18,8)$ & $20(13,0)$ & $30(19,5)$ \\
Consumo de frutas & $32(20,8)$ & $47(30,5)$ & $34(22,1)$ & $14(9,1)$ & $27(17,5)$ \\
Consumo de peixes & $42(27,3)$ & $42(27,3)$ & $22(14,3)$ & $4(2,6)$ & $44(28,5)$ \\
Consumo de ovos & $38(24,7)$ & $49(31,8)$ & $39(25,3)$ & $8(5,2)$ & $20(13,0)$ \\
Consumo de animais silvestres & $18(11,7)$ & $3(1,9)$ & $2(1,3)$ & $47(30,5)$ & $84(55,0)$ \\
\hline Alimentos não saudáveis & & & & & \\
Consumo de carne de porco & $25(16,2)$ & $3(1,9)$ & $3(1,9)$ & - & $123(79,9)$ \\
Consumo de alimentos doces & $44(28,6)$ & $37(24,0)$ & $25(16,2)$ & $16(10,4)$ & $32(20,8)$ \\
Consumo de refrigerante & $34(22,1)$ & $32(20,8)$ & $11(7,1)$ & $5(3,2)$ & $72(46,8)$ \\
Consumo de comida enlatada & $38(24,7)$ & $22(14,3)$ & $10(6,5)$ & $2(1,3)$ & $82(53,2)$ \\
Consumo de frituras & $35(22,7)$ & $57(37,0)$ & $32(20,8)$ & $7(4,5)$ & $23(15,0)$ \\
Consumo de macarrão instantâneo & $30(19,5)$ & $24(15,6)$ & $12(7,8)$ & $5(3,2)$ & $83(53,9)$ \\
Consumo de suco artificial & $33(21,4)$ & $23(15,0)$ & $16(10,4)$ & $4(2,6)$ & $78(50.6)$ \\
Consumo de pele de frango & $18(11,7)$ & $8(5,2)$ & $2(1,3)$ & - & $126(81,8)$ \\
\hline
\end{tabular}

- Corresponde a zero. Fonte: Dados da pesquisa, 2018. 
Com relação ao consumo de alimentação saudável e não saudável, a pesquisa de Mendes et al. (2016) com 155 estudantes dos três cursos de saúde da Universidade de Pernambuco mostrou que, para o consumo de frutas, os estudantes apresentaram inadequada ingestão deste grupo de alimentos $(72,9 \%)$, porém o consumo de verduras e legumes $(87,1 \%)$ esteve adequado, assim como o consumo de carnes e ovos $(78,06 \%)$. No que diz respeito às práticas alimentares, a maioria evita o consumo de frituras, embutidos ou doces $(69,0 \%)$, não adiciona sal nas refeições $(94,2 \%)$ e não troca refeições por lanches $(50,3 \%)$, o que reforça os resultados desta investigação. O estudo de Crepaldi BVC et al. (2016), que avalia a prevalência de fatores de risco para doenças crônicas entre universitários, realizado com 379 discentes de uma universidade pública do município de Uberlândia, Minas Gerais, Brasil, concluiu que os estudantes, em contraste com esta investigação, fazem baixo consumo de frutas $(76,7 \%)$, baixo consumo de feijão $(25,0 \%)$, consumo de carnes com excesso de gordura (62,5\%), ingestão excessiva de alimentos embutidos (10,8\%), e, em concordância, ingestão excessiva de alimentos doces $(26,1 \%)$ e de comidas açucaradas $(21,1 \%)$.

Quando os estudantes da presente investigação foram questionados sobre quantos dias da semana costumam trocar o almoço ou o jantar por sanduíches, salgados, pizzas ou outros lanches, 57 (37,0\%) afirmam que quase nunca fazem isso. Com relação ao consumo de sal, quando indagados acerca do que acham da soma da comida preparada na hora e os alimentos industrializados, a maior parte respondeu que é adequada $(53,9 \%)$.

Esse resultado vai de encontro ao do estudo realizado por Mendes MLM et al. (2016), em que, referente às práticas alimentares, a maioria dos participantes afirmam não adicionar sal nas refeições (94,2\%) e concordam quando se refere a não substituir refeições por lanches (50,33\%). A investigação de Alves $E F$ (2011) com 243 estudantes matriculados em um curso de graduação em Enfermagem, em Maringá - PR, mostrou que, com relação à frequência de almoço por semana, $73,4 \%$ fazem quatro a sete refeições, e, no que se refere à frequência de lanches por semana, $45,8 \%$ fazem de uma a três vezes.

$\mathrm{Na}$ presente pesquisa, com relação aos hábitos de vida, 59 (38,3\%) acadêmicos consomem bebida alcoólica e $13(8,4 \%)$ são tabagistas. Quando questionados sobre realização de atividade física, 73 (47,4\%) responderam que costumam praticar exercício físico regularmente, destes $23(31,5 \%)$ praticam de um a dois dias por semana e $42(57,5 \%)$ em 60 minutos ou mais.

Na pesquisa realizada por Leite JCA et al. (2016) com uma amostra de 90 acadêmicos de enfermagem de uma instituição privada de Montes Claros - MG, observou-se que 2,3\% dos entrevistados consomem bebida alcoólica de forma abusiva, enquanto $97,7 \%$ consomem ocasionalmente. Na análise de Castro IPP et al. (2015), 62\% dos participantes praticam exercício físico, destes, a maior parte $(33,0 \%)$ os pratica em uma frequência de três a quatro vezes por semana, $19 \%$ não os praticam e $19 \%$ já praticaram.

\section{Características da condição de saúde}

O Gráfico 1 apresenta a caracterização dos acadêmicos de enfermagem relacionada à condição de saúde.

Gráfico 1 - Condições de saúde autorrelatadas dos estudantes de enfermagem. Coari, Amazonas, 2018.

\section{Condições de saúde autorrelatadas}

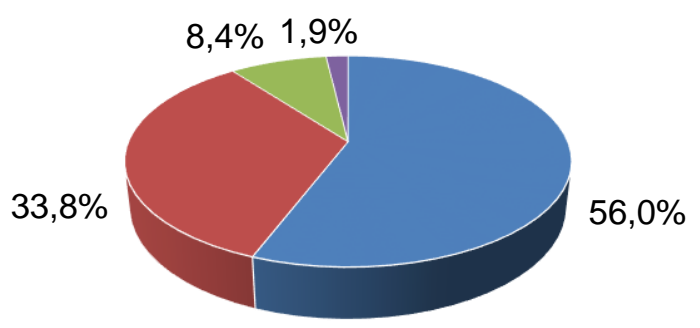

- Boa

- Regular

- Muito boa

- Ruim/Muito ruim

Fonte: Dados da pesquisa, 2018. 
No que tange ao acometimento por alguma doença, a maior parte respondeu não possuir nenhum tipo de doença $(79,9 \%)$, alguns foram diagnosticados com pressão alta $(5,8 \%)$ e colesterol elevado $(12,3 \%)$. Relacionado aos casos de diabetes na família, 45,5\% responderam sim e, destes, o maior número de casos ocorre nos avós (47,2\%).

Na investigação de Monteiro LZ et al. (2018), realizada com 286 estudantes universitários do curso de farmácia de uma instituição de ensino superior privada do Distrito Federal, em relação à autopercepção de saúde, entre as opções excelente, muito boa, boa, regular e ruim, os participantes do estudo consideram a própria saúde boa (56,0\%). Já no que diz respeito a possuir algum tipo de doença, a análise de Moreira ERA e Benito LAO, (2015) mostra que, dos 70 estudantes de enfermagem participantes da pesquisa, a maioria absoluta não possui nenhum tipo de doença $(97,2 \%)$.

Quanto à pressão arterial, Mariano KGTS et al. (2014), ao investigarem o risco para o desenvolvimento de síndrome metabólica e doença cardiovascular em 41 acadêmicos de cursos da saúde, encontraram 14,6\% dos indivíduos com pressão arterial sistêmica acima dos valores de referência e com hipertrigliceridemia, 19,5\% com hipercolesterolemia e 21,9\% dos indivíduos possuem familiar com DM2.

Com relação ao IMC, dos 154 estudantes, a maior parte está classificada como eutrófica, ou seja, dentro dos padrões normais de peso segundo a Organização Mundial da Saúde (OMS, 2000), que estabelece a seguinte classificação: baixo peso <18.50; eutrófico 18-24,99; sobrepeso 25,00-29,99; obesidade grau I 30,00-34,99; obesidade grau II 35,00-39,99; e obesidade grau III $\geq 40,00$.

A pesquisa de Pires CGS e Mussi FC (2016), realizada com 154 graduandos de enfermagem, em uma Escola de Enfermagem de uma Universidade Federal Pública, em Salvador, Bahia, constatou que há um terço de adultos jovens com excesso de peso, uma vez que $26,0 \%$ dos graduandos apresentam sobrepeso e 4,5\%, obesidade I e II.

\section{Fatores de risco modificáveis e não modificáveis}

Na Tabela 3, pode-se observar a caracterização dos acadêmicos quanto aos fatores de risco modificáveis e não modificáveis para o desenvolvimento de DM.

Tabela 3 - Fatores de risco modificáveis e não modificáveis para o desenvolvimento de DM nos estudantes de enfermagem do Instituto de Saúde e Biotecnologia da Universidade Federal do Amazonas. Coari, Amazonas, Brasil, 2018.

\begin{tabular}{lccc}
\hline Fatores de risco modificáveis & $\begin{array}{c}\text { Feminino } \\
\mathbf{N}(\%)\end{array}$ & $\begin{array}{c}\text { Masculino } \\
\mathbf{N}(\%)\end{array}$ & $\begin{array}{c}\text { Total } \\
\mathbf{N}(\%)\end{array}$ \\
\hline Não praticam atividade física & $69(44,8)$ & $12(7,8)$ & $81(52,6)$ \\
Etilismo & $38(24,7)$ & $21(13,6)$ & $59(38,3)$ \\
Consumo de sal (Alto e muito alto) & $39(25,3)$ & $12(7,8)$ & $51(33,1)$ \\
Consumo de alimentos doces (todos os dias) & $16(10,3)$ & $0(0,00)$ & $16(10,3)$ \\
Tabagismo & $8(5,2)$ & $5(3,2)$ & $13(8,4)$ \\
Consumo de frituras (todos os dias) & $6(3,9)$ & $1(0,6)$ & $7(4,5)$ \\
\hline Fatores de risco não modificáveis & & & \\
História Familiar com DM & $54(35,0)$ & $16(10,3)$ & $70(45,3)$ \\
Colesterol alto (dislipidemia) & $15(9,7)$ & $4(2,6)$ & $19(12,3)$ \\
Hipertensão Arterial Sistêmica (HAS) & $7(4,5)$ & $2(1,2)$ & $9(5,8)$ \\
\hline
\end{tabular}

Fonte: Dados da pesquisa, 2018.

Com relação aos fatores de risco modificáveis, este estudo identificou predominantemente o sedentarismo, em que a maioria dos participantes afirma que não pratica atividade física $(52,6 \%)$. É importante destacar que o ambiente dos universitários não oferece os meios adequados para a prática de atividades físicas, uma vez que não dispõe de uma área poliesportiva. Quanto ao consumo de bebida alcoólica, 59 (38,3\%) acadêmicos consomem bebidas alcoólicas e $51(33,1 \%)$ consideram seu consumo de sal alto ou muito alto. 
Quanto aos fatores de risco não modificáveis, predomina a hereditariedade, em que mais da metade dos participantes afirmam possuir no histórico familiar casos de DM $(57,8 \%)$, e o colesterol alto apresenta-se como o segundo fator de risco mais importante neste estudo (12,3\%), já a hipertensão arterial sistêmica está presente em apenas $5,8 \%$ dos participantes.

Estudo de Lima ACS et al. (2014), realizado com 702 universitários de Fortaleza, CE, Brasil, constatou que, para os 106 estudantes da área das ciências da saúde participantes da pesquisa, o fator de risco predominante foi o sedentarismo $(75,2 \%)$, seguido de excesso de peso $(12,3 \%)$, glicemia elevada $(6,6 \%)$ e hipertensão arterial (2,8\%). Em estudo de Patrício $R$ et al. (2015), realizado com 362 estudantes do Instituto Politécnico de Bragança, $61,8 \%$ dos participantes referem possuir familiares com diabetes.

\section{Classificação do risco para desenvolvimento de Diabetes Mellitus e associação com as variáveis do estudo}

A Tabela 4 demonstra a caracterização dos estudantes de enfermagem segundo as variáveis do instrumento "Are you at risk for type 2 diabetes?".

Tabela 4 - Itens do teste de risco de Diabetes Mellitus em estudantes Enfermagem do Instituto de Saúde e Biotecnologia da Universidade Federal do Amazonas. Coari, Amazonas, Brasil, 2018.

\begin{tabular}{lll}
\hline Variáveis & $\mathbf{N}$ & $\%$ \\
\hline Idade (anos) & 153 & 99,4 \\
$<40$ & 1 & 0.6 \\
$\geq 40$ & 105 & 68,2 \\
\hline Sexo & 49 & 31,8 \\
Mulher & & \\
Homem & 153 & 99,4 \\
\hline Diabetes Gestacional & 1 & 0,6 \\
Não & & \\
Sim & 131 & 85,1 \\
\hline Mãe, pai, irmã ou irmão com diabetes? & 23 & 14,9 \\
Não & & \\
Sim & 145 & 94,2 \\
\hline Pressão Arterial & 9 & 5,8 \\
Não & & 47,4 \\
Sim & & 52,6 \\
\hline Fisicamente ativo & 73 & 87,7 \\
Sim & 81 & 11,0 \\
Não & & 1,3 \\
\hline Status de Peso & 135 & \\
1 & 17 & 2 \\
2 & & \\
3 & 23 & \\
\hline
\end{tabular}

Fonte: Dados da pesquisa, 2018.

É importante ressaltar que o instrumento "Are you at risk for type 2 diabetes?" apresenta um escore mínimo de um e máximo de oito pontos, sendo que igual ou menor a quatro pontos significa sem risco e igual ou superior a cinco remete a risco de desenvolver diabetes. Nesta investigação, observou-se que mais da metade dos acadêmicos somaram dois pontos $(51,3 \%)$, outra parcela somou três pontos $(24,0 \%)$ e apenas $1,9 \%$ apresentou cinco pontos, ou seja, dos 154 acadêmicos, apenas três apresentam risco para desenvolver DM2. 
Em busca de fatores de risco para DM2, Magalhães AT et al. (2015) utilizaram o mesmo instrumento empregado neste estudo e apontaram que apenas 16 (1,23\%) de 1299 estudantes universitários apresentou risco de DM do tipo 2. Ainda dos resultados obtidos nesta investigação, constatou que $518(39,9 \%)$ eram homens, $1263(97,2 \%)$ tinham idade inferior a 40 anos de idade, $234(18,0 \%)$ tinham história familiar de DM, $77(5,9 \%)$ com diagnóstico de hipertensão arterial sistêmica, $536(41,2 \%)$ realizam atividade física, 599 $(46,1 \%)$ pontuaram para o status de peso, porém apenas $56(4,5 \%)$ pontuaram com dois pontos.

\section{Associação do risco para desenvolvimento de DM2 com as variáveis do estudo}

A Tabela 5 destaca a análise entre as variáveis dos itens do instrumento chamado de "Are you at risk for type 2 diabetes?" utilizado neste estudo sobre riscos para desenvolvimento de Diabetes Mellitus do tipo 2 associado a classificação de calouro e veterano dos participantes do estudo.

Tabela 5 - Análise da associação dos riscos para o desenvolvimento de Diabetes Mellitus do tipo 2 com a classificação de calouro e veterano dos participantes do estudo Coari, Amazonas, Brasil, 2018.

\begin{tabular}{|c|c|c|c|c|c|}
\hline \multirow{3}{*}{ Riscos de DM 2} & \multicolumn{5}{|c|}{ Universitários } \\
\hline & \multicolumn{2}{|c|}{ Calouros $(\mathrm{N}=43)$} & \multicolumn{2}{|c|}{ Veteranos $(\mathrm{N}=111)$} & \multirow{2}{*}{$\mathbf{p}^{*}$} \\
\hline & $\mathbf{N}$ & $\%$ & $\mathbf{N}$ & $\%$ & \\
\hline \multicolumn{6}{|l|}{ Homens } \\
\hline Não & 31 & 29,5 & 74 & 70,5 & \multirow{2}{*}{$0,517^{*}$} \\
\hline Sim & 12 & 24,5 & 37 & 75,5 & \\
\hline \multicolumn{6}{|c|}{ Diabetes Gestacional } \\
\hline Não & 43 & 28,1 & 110 & 71,9 & \multirow{2}{*}{$0,532^{* *}$} \\
\hline Sim & 0 & 0,0 & 1 & 100,0 & \\
\hline \multicolumn{6}{|l|}{ Familiaridade } \\
\hline Não & 38 & 29,0 & 93 & 71,0 & \multirow{2}{*}{$0,474^{*}$} \\
\hline Sim & 5 & 21,7 & 18 & 78,3 & \\
\hline \multicolumn{6}{|c|}{ Hipertensão Arterial Sistêmica (HAS) } \\
\hline Não & 41 & 28,3 & 104 & 71,7 & \multirow{2}{*}{$0,694^{* *}$} \\
\hline Sim & 2 & 22,2 & 7 & 77,8 & \\
\hline \multicolumn{6}{|l|}{ Inatividade física } \\
\hline Não & 41 & 28,1 & 105 & 71,9 & \multirow{2}{*}{$0,850^{* *}$} \\
\hline Sim & 2 & 25,0 & 6 & 75,0 & \\
\hline \multicolumn{6}{|l|}{ Risco do peso } \\
\hline $\operatorname{Sim}$ & 41 & 30,1 & 95 & 69,9 & \multirow[t]{2}{*}{$0,091^{* *}$} \\
\hline Não & 2 & 11,1 & 16 & 88,9 & \\
\hline
\end{tabular}

*Teste qui-quadrado de Pearson ** Teste exato de Fisher.

Fonte: Dados da pesquisa, 2018.

Verificou-se que o estudo não apresentou nenhuma associação estatística com as variáveis analisadas. O estudo de Lima ACS et al. (2014) aponta que, entre a maioria dos novatos, está presente o sedentarismo $(71,9 \%)$, enquanto que, entre os veteranos, predomina o sobrepeso $(24,4 \%)$ e a obesidade $(7 \%)$, o que vai ao encontro do presente estudo, no qual, entre os novatos, os fatores de risco predominantes são inatividade física e risco de peso, enquanto que, para os veteranos, acrescenta-se a hereditariedade. 


\section{CONCLUSÃO}

Os principais fatores de risco modificáveis entre os acadêmicos são: estilo de vida sedentário, etilismo, consumo de sal e ainda um padrão de sono inadequado que provoca o estresse e, como consequência, maior vulnerabilidade para o acometimento de doenças. E não modificáveis: história familiar com diabetes, colesterol alto e hipertensão arterial sistêmica. Dessa forma, este estudo constatou que, apesar de ocorrer uma transição no estilo de vida do estudante ao ingressar na vida acadêmica, não influencia de forma significativa o desenvolvimento de DM2. Contudo, apesar da baixa incidência de DM2 entre universitários, é importante adotar um estilo de vida saudável, uma vez que há outras DCNTs, para as quais há suscetibilidade por parte dos graduandos.

\section{REFERÊNCIAS}

1. ALVES EF. Estilo de vida de estudantes de graduação em enfermagem de uma instituição do sul do Brasil / Lifestyle of undergraduate nursing students of an institution from southern Brazil, 2011.

2. BRASIL. Ministério da Saúde. Secretaria de Vigilância em Saúde. Departamento de Análise de Situação de Saúde. Plano de ações estratégicas para o enfrentamento das doenças crônicas não transmissíveis (DCNT) no Brasil 2011-2022. série b. t ed. Brasília: Ministério da Saúde, 2011. v. 1

3. BRASIL. Ministério da Saúde. Secretaria de Vigilância em Saúde. Vigitel Brasil 2015: vigilância de fatores de risco e proteção para doenças crônicas por inquérito telefônico: estimativas sobre frequência e distribuição sociodemográfica de fatores de risco e proteção para doenças crônicas nas capitais dos 26 Estados, Vigitel. 2016. 162 p.

4. BUBLITZ S, et al. Associação entre estresse e características sociodemográficas e acadêmicas de estudantes de enfermagem. Texto \& Contexto Enfermagem, 2016; 25(4): 1-7

5. BARLEM JGT, et al. Síndrome de Burnout entre estudantes de graduação em enfermagem de uma universidade pública. Revista Latino-Americana de Enfermagem 2014; 22(6): 934-41.

6. CASTRO YPP, et al. Fatores de risco para hipertensão arterial em acadêmicos de enfermagem de uma universidade. Revista Interdisciplinar, 2015; 8(3): 128-134.

7. CREPALDI BVC, et al. Elevada prevalência de fatores de risco para doenças crônicas entre universitários High prevalence of risk factors for chronic diseases among university students. Ciencia \& Saúde, 2016; 9(3): 135-143.

8. DUNCAN BB, et al. Doenças Crônicas Não Transmissíveis no Brasil: prioridade para enfrentamento e investigação. Revista de Saúde Pública, 2012; 46: 126-134.

9. GONÇALVES A, et al. Insónia nos estudantes de enfermagem em ensino clínico. Revista Portuguesa de Enfermagem de Saúde Mental, 2016; 3: 73-8.

10. IBGE. Instituto Brasileiro de Geografia e Estatística. Censo Demográfico 2010 - Características gerais da população, religião e pessoas com deficiência. 2012; 1:1-30.

11. IDF. Diabetes Atlas. 7. ed. Brussels: International Diabetes Federation, 2015.

12. INEP, Instituto Nacional de Estudos e Pesquisas Educacionais Anísio Teixeira. Questionário do estudante 2016, Ministério da Educação. 2016;1-8.

13. LEITE JCA, et al. Consumo de álcool entre os acadêmicos de Enfermagem 50. Revista Bionorte, 2016; 5: 50-58.

14. LIMA ACS, et al. Fatores de risco para diabetes mellitus tipo 2 em universitários: associação com variáveis sociodemográficas. Revista Latino-Americana de Enfermagem, 2014; 22(3): 484-490.

15. LOURENÇO CLM, et al. Comportamento sedentário em estudantes Universitários. Revista Brasileira de Atividade Física \& Saúde, 2016; 21(1): 67-77.

16. MAGALHÃES AT, et al. Avaliação do risco de desenvolver diabetes mellitus tipo 2 em população universitária. Revista Brasileira em Promoção da Saúde, 2015; 28(1): 5-15.

17. MALETTA, CHM. Epidemiologia das Doenças Crônicas. $3^{a}$ ed. Belo Horizonte: Coopmed, 2016.

18. MARIANO KGTS, et al. Identificação de fatores de risco para o desenvolvimento de síndrome metabólica e doença cardiovascular em estudantes universitários. Cadernos da Escola de Saúde, Curitiba, 2014; 2: 50-62.

19. MARINHO N, et al. Risco para diabetes mellitus tipo 2 e fatores associados. Acta Paulista Enfermagem, 2013; 26(6): 569-74.

20. MENDES MLM, et al. Hábitos alimentares e atividade física de universitários da área de saúde do município de Petrolina-PE. Tempus, actas de saúde coletiva, 2016; 10(2): 205-217.

21. MONTEIRO LZ, et al. Uso de tabaco e álcool entre acadêmicos da saúde. Revista Brasileira em Promoção da Saúde, 2018; 31(1): $1-9$.

22. MOREIRA ERA, BENITO LAO. Segurança alimentar de graduandos em enfermagem. Monografia, Centro Universitário de Brasília, 2015.

23. WHO. World Health Organization. Technical Report Series Obesity: preventing and managing the global epidemic Report of a WHO Consultation. 2000.

24. PAIXÃO CC, et al. Desafios no ensino de computação: um estudo da relação entre perfil psicológico de alunos e evasão. WEI XXI Workshop sobre Educação em Computação,2013; 720-29.

25. PATRÍ́CIO R, et al. Diabetes mellitus na comunidade do Instituto Politécnico de Bragança: caracterização e conhecimentos. Escola Superior de Tecnologia da Saúde do Instituto Politécnico do Porto (ESTSP-IPP) 2015; 1.

26. PIRES CGS, MUSSI FC. Excesso de peso em universitários ingressantes e concluintes de um curso de enfermagem. Escola Anna Nery Revista de Enfermagem, Rio de Janeiro. 2016; 20(4).

27. SANTOS JDS, et al. Avaliação para riscos cardiovasculares em estudantes de enfermagem. Revista Mineira de Enfermagem, 2015;19(4): 842-7.

28. SCHMIDT MI, et al. Doenças crônicas não transmissíveis no Brasil: carga e desafios atuais. The Lancet, 2011; 377(9781): 194961.

29. SEHNEM D, et al. A sexualidade na formação acadêmica do enfermeiro. Escola Anna Nery Revista de Enfermagem, Rio de Janeiro. 2013; 17(1): 90-6.

30. SUDARTH BE, et al. Brunner \& Suddarth's: Tratado de Enfermagem médico-cirúrgica. 13. ed. RJ: Guanabara Koogan, 2016. 